\title{
A tight analysis of Brown-Baker-Katseff sequences for online strip packing
}

\author{
W. Kern · J.J. Paulus
}

Published online: 24 February 2012

(C) The Author(s) 2012. This article is published with open access at Springerlink.com

\begin{abstract}
We study certain adversary sequences for online strip packing which were first designed and investigated by Brown, Baker and Katseff (Acta Inform. 18:207225) and determine the optimal competitive ratio for packing such Brown-BakerKatseff sequences online. As a byproduct of our result, we get a new lower bound of $\rho \geq 3 / 2+\sqrt{33} / 6 \approx 2.457$ for the competitive ratio of online strip packing.
\end{abstract}

Keywords Online algorithm $\cdot$ Strip packing $\cdot$ Competitive ratio

\section{Introduction}

In the two-dimensional strip packing problem a number of rectangles have to be packed without rotation or overlap into a strip such that the height of the strip used is minimum. The width of the rectangles is bounded by 1 and the strip has width 1 and infinite height. Baker et al. (1980) show that this problem is NP-hard.

We study the online version of this packing problem. In the online version the rectangles are given to the online algorithm one by one from a list, and the next rectangle is given as soon as the current rectangle is irrevocably placed into the strip. To evaluate the performance of an online algorithm we employ competitive analysis. For a list of rectangles $L$, the height of a strip used by online algorithm $A$ and by the optimal solution is denoted by $A(L)$ and $O P T(L)$, respectively. The optimal solution is not restricted in any way by the ordering of the rectangles in the list. Competitive analysis measures the absolute worst-case performance of online algorithm $A$ by its competitive ratio $\sup _{L}\{A(L) / O P T(L)\}$ (cf. Pruhs et al. 2004).

W. Kern $(\varangle)$

Enschede, The Netherlands

e-mail: kern@math.utwente.nl

J.J. Paulus

CQM BV, P.O. Box 414, 5600 AK Eindhoven, The Netherlands 
Regarding the upper bound on the competitive ratio for online strip packing, recent advances have been made by Ye et al. (2009) and Hurink and Paulus (2008). Independently they show that a modification of the well-known shelf algorithm yields an online algorithm with competitive ratio $7 / 2+\sqrt{10} \approx 6.6623$. We refer to these two papers for a more extensive overview of the literature.

In the early 80's, Brown et al. (1982) derived a lower bound $\rho \geq 2$ on the competitive ratio of any online algorithm by constructing certain (adversary) sequences in a fairly straightforward way (cf. Sect. 2). These sequences were further studied by Johannes (2006) and Hurink and Paulus (2008), who derived improved lower bounds of 2.25 and 2.43, resp. (Both results are computer aided and presented in terms of online parallel machine scheduling, a closely related problem.) The paper of Hurink and Paulus (2008) also presents an upper bound of $\rho \leq 2.5$ for packing such "BrownBaker-Katseff sequences". The purpose of our present paper is to close the gap between 2.43 and 2.5 by presenting a tight analysis, showing that Brown-Baker-Katseff sequences can be packed online with competitive ratio $\rho=3 / 2+\sqrt{33} / 6$ and that this is best possible. As a byproduct, we obtain a new lower bound $\rho \approx 2.457$ for online strip packing.

The result of this paper has been presented at the CTW 2010 (cf. Kern 2010). Meanwhile, in a joint work with R. Harren, we tried to analyze a modified version of Brown-Baker-Katseff sequences, yielding a slightly better lower bound (but no exact analysis as here), cf. Harren and Kern (2011).

\section{The instance construction}

In this section we describe the construction of Brown-Baker-Katseff sequences $L_{n}$ according to Brown et al. (1982). In addition, we present an online algorithm for packing the sequences $L_{n}$ online with ratio $\hat{\rho}=3 / 2+\sqrt{33} / 6$. For convenience, let throughout this note $\hat{\rho}=3 / 2+\sqrt{33} / 6$.

We define $L_{n}$ as the list of rectangles $\left(p_{0}, q_{1}, p_{1}, q_{2}, p_{2}, \ldots, q_{n}, p_{n}\right)$, where $p_{i}$ denotes a rectangle of height $p_{i}$ and negligible width (no more than $1 /(n+1)$ ), and $q_{i}$ denotes a rectangle of height $q_{i}$ and width 1 . The rectangle heights are defined such that, when the items are packed online, each item must be packed on top of the preceding ones. More precisely, we let

$$
\begin{aligned}
p_{0} & =1, \\
p_{i} & =\beta_{i-1} p_{i-1}+p_{i-1}+\alpha_{i} p_{i}+\epsilon \quad \forall i \geq 1, \\
q_{1} & =\beta_{0} p_{0}+\epsilon, \\
q_{i} & =\max \left\{\alpha_{i-1} p_{i-1}, q_{i-1}, \beta_{i-1} p_{i-1}\right\}+\epsilon \quad \forall i \geq 2,
\end{aligned}
$$

where $\alpha_{i} p_{i}$ and $\beta_{i} p_{i}$ are distances the online algorithm has placed between earlier rectangles, and $\epsilon$ is a small positive value. The value $\alpha_{i} p_{i}$ denotes the vertical distance between rectangles $p_{i-1}$ and $q_{i}$, and the value $\beta_{i} p_{i}$ denotes the vertical distance between $q_{i}$ and $p_{i}$. This is illustrated in Fig. 1 . The values $\alpha_{i}$ and $\beta_{i}$ completely characterize the behavior of the online algorithm when processing $L_{n}$. For consistency we define in addition $\alpha_{0}=0$. 
Fig. 1 Online and optimal packing of $L_{2}$

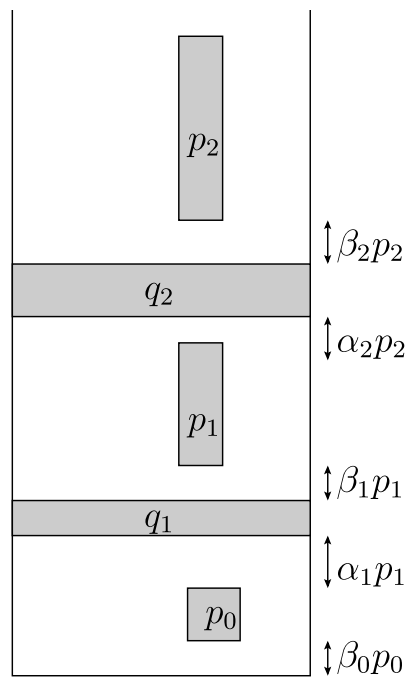

Packing by the online algorithm

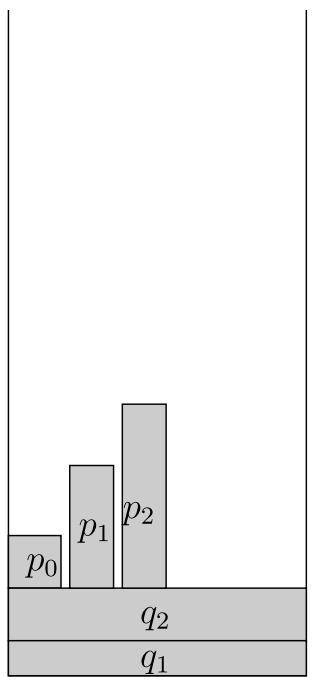

An optimal packing

By definition of the rectangles' heights and widths, an online algorithm can only pack the rectangles one above the other in the same order as the rectangles appear in the list $L_{n}$. An optimal offline packing is obtained by first packing the rectangles $q_{i}$ on top of each other and then pack all $p_{i}$ next to each other on top of the $q$-rectangles. The sole function of the positive term $\epsilon$ is to ensure this structure on any online packing. From now on we assume that $\epsilon$ is small enough to be omitted from the analysis.

We start with the (simpler) upper bound:

Theorem 1 Each list $L_{n}$ can be packed online with competitive ratio $\hat{\rho}=\frac{3}{2}+\frac{\sqrt{33}}{6}$.

Proof Consider the online algorithm $A$ that chooses $\beta_{0}=\hat{\rho}-1, \alpha_{2}=1 /(\hat{\rho}-1)$, and all other gaps equal to 0 . So $p_{0}=1, q_{1}=q_{2}=\hat{\rho}-1, p_{1}=\hat{\rho}$, and $p_{2}$ can be computed from $p_{2}=p_{1}+\alpha_{2} p_{2}$ : We get $p_{2}=\hat{\rho} /\left(1-\frac{1}{\hat{\rho}-1}\right)=\frac{(\hat{\rho}-1) \hat{\rho}}{\hat{\rho}-2}$, or $p_{2}=$ $(\hat{\rho}-1)(3 \hat{\rho}-2)=4 \hat{\rho}-2$ by our choice of $\hat{\rho}$.

We claim that the resulting algorithm is $\hat{\rho}$-competitive when presented with $L_{n}$.

- After packing $p_{0}=1$ we have $A\left(L_{0}\right)=\hat{\rho}$ and $O P T\left(L_{0}\right)=1$. Thus, the competitive ratio is exactly $\hat{\rho}$ at this point.

- After packing rectangle $q_{1}$ the online and optimal packing increase by the same amount. Thus the competitive ratio decreases.

- After $p_{1}=\hat{\rho}$ we have $A\left(L_{1}\right)=\beta_{0} p_{0}+p_{0}+q_{1}+p_{1}=\hat{\rho}-1+1+\hat{\rho}-1+\hat{\rho}$, while $\operatorname{OPT}\left(L_{1}\right)=q_{1}+p_{1}=2 \rho-1$. Hence $A\left(L_{1}\right) / O P T\left(L_{1}\right)=(3 \hat{\rho}-1) /(2 \hat{\rho}-1)<\hat{\rho}$.

- After $q_{2}$ we have $A\left(L_{1} q_{2}\right)=A\left(L_{1}\right)+q_{2}+\alpha_{2} p_{2}=3 \hat{\rho}-1+\hat{\rho}-1+3 \hat{\rho}-2=$ $7 \hat{\rho}-4$, while $\operatorname{OPT}\left(L_{1} q_{2}\right)=p_{1}+q_{1}+q_{2}=3 \rho-2$. So $A\left(L_{1} q_{2}\right) / O P T\left(L_{1} q_{2}\right)=$ $(7 \hat{\rho}-4) /(3 \hat{\rho}-2)=\hat{\rho}$. Again the competitive ratio is exactly $\hat{\rho}$ at this point (by definition of $\hat{\rho})$. 
- After $p_{2}$ we have $A\left(L_{2}\right)=A\left(L_{1} q_{2}\right)+p_{2}=7 \hat{\rho}-4+4 \hat{\rho}-2=11 \hat{\rho}-6$, while $\operatorname{OPT}\left(L_{2}\right)=q_{1}+q_{2}+p_{2}=2 \hat{\rho}-2+4 \hat{\rho}-2=6 \hat{\rho}-4$. Thus $A\left(L_{2}\right) / O P T\left(L_{2}\right)=$ $(11 \hat{\rho}-6) /(6 \hat{\rho}-4)<\hat{\rho}$.

- For $i \geq 3$ there are no more gaps introduced by online algorithm $A$. In particular, $p_{2}=p_{3}=p_{4}=\cdots=(\hat{\rho}-1)(3 \hat{\rho}-2)$ and $q_{i}=\alpha_{2} p_{2}=3 \hat{\rho}-2$ for all $i \geq 3$. When packing $q_{i}$, the online and optimal packing increase by the same amount and, thus, $\rho$-competitiveness is not violated. After packing $p_{i+1}$, however, we have $O P T\left(L_{i+1}\right)=O P T\left(L_{i}\right)+q_{i+1}$ and $A\left(L_{i+1}\right)=A\left(L_{i}\right)+q_{i+1}+p_{i+1}=A\left(L_{i}\right)+$ $\hat{\rho} q_{i+1}$. The height of the online packing grows exactly $\hat{\rho}$ times as fast as the optimal packing.

So, online algorithm $A$ is $\hat{\rho}$-competitive for the list of rectangles $L_{n}$.

\section{Lower bound on the competitive ratio}

In this section we prove a lower bound of $\hat{\rho}=3 / 2+\sqrt{33} / 6$ on the competitive ratio for online packing Brown-Baker-Katseff sequences - and hence for online strip packing in general. The outline of the proof is as follows.

Assume that there exists a $\rho$-competitive online algorithm $A$ with $\rho<\hat{\rho}$. We present this algorithm with the list $L_{n}$, with $n$ arbitrarily large. To obtain a contradiction we define a potential function $\Phi_{i}$ on the state of the online packing after packing rectangle $p_{i}$. We argue that this potential function is both bounded from below and that it decreases to $-\infty$, giving us the required contradiction.

After packing the rectangle $p_{i}$, we measure with $\gamma_{i}$ how much online algorithm $A$ improves upon the $\rho$-competitiveness bound: We define $\gamma_{i}$ through

$$
A\left(L_{i}\right)+\gamma_{i} p_{i}=\rho O P T\left(L_{i}\right) .
$$

The potential function $\Phi_{i}$ is defined (after packing rectangle $p_{i}$ ) by

$$
\Phi_{i}:=\frac{\gamma_{i}+\beta_{i}-(\rho-2) \alpha_{i}}{1-\alpha_{i}}
$$

We admit that the potential function looks rather involved. Its exact form is simply motivated by the technical analysis that follows. In particular, the potential function is designed such that a certain "shift invariance" (cf. Lemma 2 below) holds, which helps a lot in simplifying the analysis. (Yet, in Harren and Kern 2011 we analyze "extended" Brown-Baker-Katseff sequences with a simpler potential $\Phi_{i}=\gamma_{i}+\beta_{i}$ and considerable technical effort.)

The values of $\alpha_{i}$ and $\beta_{i}$ are nonnegative by definition and $\gamma_{i}$ is nonnegative by the $\rho$-competitiveness of online algorithm $A$. Observe that shifting $p_{i}$, say, upward, increases $\beta_{i}$ and decreases $\gamma_{i}$ by the same amount, so that shifting $p_{i}$ has actually no effect on $\Phi_{i}$. In Lemma 2 we will see that the same holds w.r.t. shifting $q_{i}$. Thus the decisions of the online algorithm in "phase $i$ " have no effect on $\Phi_{i}$, but rather on subsequent potential values. (This phenomenon is observed also elsewhere, cf., e.g. Fuchs et al. 2005 or Harren and Kern 2011.) The crucial result is Lemma 6, stating that $\rho<\hat{\rho}$ forces a significant decrease of the potential in each step. Thus 
$\Phi_{i+1} \rightarrow-\infty$ for $i \rightarrow \infty$. On the other hand, in Lemma 5 we show among other things that $\alpha_{i}<1 /(\hat{\rho}-1)$. As a consequence, $\Phi_{i}>-1$ for all $i$. This contradiction finally implies $\rho \geq \hat{\rho}$, the main result of this note:

Theorem 2 The best possible ratio for packing Brown-Baker-Katseff sequences is $\hat{\rho}=3 / 2+\sqrt{33} / 6$. This value also provides a lower bound for online strip packing.

The remainder of this note is concerned with the proofs of the afore mentioned lemmata. We start by providing some basic properties (Lemmas 1 and 2 below) of the potential function $\Phi_{i}$. In the following, $\rho$ is assumed to be suitably close to $\hat{\rho}$ whenever this is necessary.

First observe that the following equation defines $p_{i+1}$ according to the construction rule for the list $L_{n}$. We will use it at several places during our analysis:

$$
\left(\beta_{i}+1\right) p_{i}=\left(1-\alpha_{i+1}\right) p_{i+1} .
$$

Lemma 1 If $\Phi_{i} \leq \rho-1$ then $\gamma_{i}+\beta_{i}+\alpha_{i} \leq \rho-1$.

Proof

$$
\begin{aligned}
\Phi_{i} \leq \rho-1 & \Rightarrow \gamma_{i}+\beta_{i}-(\rho-2) \alpha_{i} \leq(\rho-1)\left(1-\alpha_{i}\right) \\
& \Rightarrow \gamma_{i}+\beta_{i}+\alpha_{i} \leq \rho-1 .
\end{aligned}
$$

Lemma 2 The potential $\Phi_{i}$ is invariant under shifting $p_{i}$ and/or $q_{i}$.

Proof Shifting rectangle $p_{i}$, say, upward by one unit does not affect $\operatorname{OPT}\left(L_{i}\right)$ nor $\alpha_{i}$. Furthermore, $A\left(L_{i}\right)$ increases by one unit and, hence, $\gamma_{i} p_{i}$ decreases by one unit. At the same time $\beta_{i} p_{i}$ increases by one unit, so that $\gamma_{i}+\beta_{i}$ remains constant and $\Phi_{i}$ is invariant under shifting $p_{i}$.

To show that $\Phi_{i}$ is invariant under shifting $q_{i}$, assume w.l.o.g. that $\beta_{i}=0$, i.e., that $p_{i}$ has been shifted down to $q_{i}$, and that we shift the concatenated rectangles $q_{i} p_{i}$ simultaneously, say, upward. This causes an increase of $p_{i}$ by one unit, so $A\left(L_{i}\right)$ increases in total by 2 units. On the other hand, $O P T\left(L_{i}\right)$ increases by just one unit, so $\gamma_{i} p_{i}$ will increase by $\rho-2$ units. Clearly, $\alpha_{i} p_{i}$ increases by one unit and $\left(1-\alpha_{i}\right) p_{i}=$ $\left(1+\beta_{i-1}\right) p_{i-1}$ remains constant. Hence

$$
\Phi_{i}=\frac{\gamma_{i} p_{i}-(\rho-2) \alpha_{i} p_{i}}{\left(1-\alpha_{i}\right) p_{i}}
$$

will indeed remain constant.

Clearly, shifting $p_{i}$ and/or $q_{i}$ does have an effect on subsequent values like $p_{i+1}$ and $\Phi_{i+1}$. Furthermore, even when we are only interested in packing $L_{i}$, shifting up $p_{i}$ or shifting down the concatenated $q_{i} p_{i}$ may result in an infeasible packing. (Indeed, as we shift $q_{i} p_{i}$ down, $p_{i}$ decreases and after a while the packing may cease to be $\rho$-competitive!) As long as we are only interested in the value of $\Phi_{i}$, however, we may well shift $p_{i}$ and $q_{i}$ as we like, disregarding the competitiveness constraints. We will make extensive use of this observation further on. 


\section{Lemma 3}

$$
\Phi_{i+1}=\frac{\gamma_{i}+(\rho-1) \beta_{i}-1+(\rho-1) q_{i+1} / p_{i}}{1+\beta_{i}}
$$

Proof By Lemma 2 we can shift rectangle $p_{i+1}$ down, i.e. $\beta_{i+1}=0$. Then

$$
\begin{aligned}
\left(1-\alpha_{i+1}\right) p_{i+1} \Phi_{i+1}= & \left(\gamma_{i+1}+\beta_{i+1}-(\rho-2) \alpha_{i+1}\right) p_{i+1} \\
= & (\operatorname{Lem} .2)\left(\gamma_{i+1}-(\rho-2) \alpha_{i+1}\right) p_{i+1} \\
= & \rho O P T\left(L_{i+1}\right)-A\left(L_{i+1}\right)-(\rho-2) \alpha_{i+1} p_{i+1} \\
= & \rho\left(\operatorname{OPT}\left(L_{i}\right)+q_{i+1}+\beta_{i} p_{i}+\alpha_{i+1} p_{i+1}\right) \\
& -\left(A\left(L_{i}\right)+\alpha_{i+1} p_{i+1}+q_{i+1}+p_{i+1}\right) \\
& -(\rho-2) \alpha_{i+1} p_{i+1} \\
= & \gamma_{i} p_{i}+\rho \beta_{i} p_{i}-\left(1-\alpha_{i+1}\right) p_{i+1}+(\rho-1) q_{i+1} \\
= & { }_{(1)}\left(\gamma_{i}+(\rho-1) \beta_{i}-1\right) p_{i}+(\rho-1) q_{i+1} .
\end{aligned}
$$

By (1) we can divide the left hand side by $\left(1-\alpha_{i+1}\right) p_{i+1}$ and the right hand side by $\left(1+\beta_{i}\right) p_{i}$ to obtain the result.

Lemma 4 If $q_{i+1}=\max \left\{\alpha_{i} p_{i}, q_{i}\right\}$, then we may assume w.l.o.g. that $\beta_{i}=0$.

Proof Shifting rectangle $p_{i}$ down decreases the distance $\beta_{i} p_{i}$ and increases $\alpha_{i+1} p_{i+1}$. However, when we keep all other distances equal it does not affect $p_{j}$ with $j>i$. Due to the increase in $\alpha_{i+1} p_{i+1}$ some $q_{j}$ with $j>i$ may increase, but this is only in favor of the online algorithm since the optimal value increases by exactly the same amount. So the alternative online algorithm that schedules $p_{i}$ earlier and leaves all other distances unchanged is also feasible.

Lemma 5 For $i \geq 0, \Phi_{i} \leq \rho-1$, and for $i \geq 1, \alpha_{i} \leq 1 /(\hat{\rho}-1)$ and $q_{i} / p_{i} \leq 1(\hat{\rho}-1)$. In case $q_{i}=\alpha_{i-1} p_{i-1}$ or $q_{i}=q_{i-1}$, we even have $q_{i} / p_{i} \leq\left(1-\alpha_{i}\right) /(\hat{\rho}-1)$.

Proof By induction: The claim holds for $i=0$ since $\alpha_{0}=0$ by definition, $\gamma_{0}+\beta_{0}=$ $\rho-1$ and thus $\Phi_{0}=\rho-1$. We assume the lemma holds up to $i$, and prove it for $i+1$ by case distinction on the way the height of rectangle $q_{i+1}$ is determined. (For $i=1$, case 2 applies.)

Case 1: $q_{i+1}=\alpha_{i} p_{i}$.

By Lemma 4 we may assume $\beta_{i}=0$. By (1), this further implies $p_{i}=(1-$ $\left.\alpha_{i+1}\right) p_{i+1}$. Hence

$$
\frac{q_{i+1}}{p_{i+1}}=\frac{\alpha_{i} p_{i}}{p_{i}+\alpha_{i+1} p_{i+1}}=\frac{\alpha_{i}\left(1-\alpha_{i+1}\right) p_{i+1}}{\left(1-\alpha_{i+1}\right) p_{i+1}+\alpha_{i+1} p_{i+1}}=\left(1-\alpha_{i+1}\right) \alpha_{i} \leq \frac{1-\alpha_{i+1}}{\hat{\rho}-1}
$$

by induction.

The online algorithm $A$ is by assumption $\rho$-competitive after packing rectangle $q_{i+1}$, which means that the distance between rectangles $q_{i+1}$ and $p_{i}$ is not too large, 
i.e., $\alpha_{i+1} p_{i+1} \leq \gamma_{i} p_{i}+(\rho-1) q_{i+1}=\gamma_{i} p_{i}+(\rho-1) \alpha_{i} p_{i}$. Together with Lemma 1 this gives

$$
\begin{aligned}
\alpha_{i+1} & =\frac{\alpha_{i+1} p_{i+1}}{p_{i+1}}=\frac{\alpha_{i+1} p_{i+1}}{p_{i}+\alpha_{i+1} p_{i+1}} \leq \frac{\gamma_{i}+(\rho-1) \alpha_{i}}{1+\gamma_{i}+(\rho-1) \alpha_{i}} \\
& \leq \frac{(\rho-1)^{2}}{1+(\rho-1)^{2}}<\frac{1}{\hat{\rho}-1}
\end{aligned}
$$

for $\rho \leq \hat{\rho}$. (This upper bound for $\alpha_{i+1}$, obtained by setting $\gamma_{i}=0$ and $\alpha_{i}=\rho-1$ (cf. Lemma 1) is rather weak, but sufficient for our purposes.)

Finally, by Lemma 3, the induction assumption and Lemma 1 we get

$$
\Phi_{i+1}=\gamma_{i}-1+(\rho-1) q_{i+1} / p_{i}=\gamma_{i}-1+(\rho-1) \alpha_{i}<\gamma_{i} \leq \rho-1 .
$$

Case 2: $q_{i+1}=\beta_{i} p_{i}$.

By Lemma 1 we have $\beta_{i} \leq \rho-1$ and thus,

$$
\frac{q_{i+1}}{p_{i+1}}=\frac{\beta_{i} p_{i}}{\left(1+\beta_{i}\right) p_{i}+\alpha_{i+1} p_{i+1}} \leq \frac{\beta_{i}}{1+\beta_{i}} \leq \frac{\rho-1}{\rho}
$$

which is less than $1 /(\hat{\rho}-1)$ for $\rho \leq \hat{\rho}$.

The online algorithm $A$ is by assumption $\rho$-competitive after packing rectangle $q_{i+1}$, which means that the distance between rectangles $q_{i+1}$ and $p_{i}$ is not too large, i.e. $\alpha_{i+1} p_{i+1} \leq \gamma_{i} p_{i}+(\rho-1) q_{i+1}=\gamma_{i} p_{i}+(\rho-1) \beta_{i} p_{i}$. This, together with $\beta_{i}+\gamma_{i} \leq \rho-1$ (by Lemma 1 ) gives

$$
\begin{aligned}
\alpha_{i+1} & =\frac{\alpha_{i+1} p_{i+1}}{p_{i+1}}=\frac{\alpha_{i+1} p_{i+1}}{\left(1+\beta_{i}\right) p_{i}+\alpha_{i+1} p_{i+1}} \leq \frac{\gamma_{i}+(\rho-1) \beta_{i}}{\left(1+\beta_{i}\right)+\gamma_{i}+(\rho-1) \beta_{i}} \\
& =\frac{\gamma_{i}+(\rho-1) \beta_{i}}{1+\gamma_{i}+\rho \beta_{i}} \leq \frac{(\rho-1)}{1+(\rho-1)}<\frac{1}{\hat{\rho}-1}
\end{aligned}
$$

for $\rho \leq \hat{\rho}$. (In the upper bound computation for $\alpha_{i}$ above we assumed that $\gamma_{i}$ is as large as possible and $\beta_{i}$ is as small as possible. This is justified by the fact that $\frac{\rho-1}{\rho}<\frac{1}{\hat{\rho}-1}$.)

For the potential, Lemma 3 gives

$$
\Phi_{i+1}=\frac{\gamma_{i}+2(\rho-1) \beta_{i}-1}{1+\beta_{i}} \leq \frac{2(\rho-1)^{2}-1}{\rho},
$$

which is strictly less than $\rho-1$ for $\rho$ sufficiently close to $\hat{\rho}$. (Again, for the last inequality in (3), note that increasing $\beta_{i}$ as much as possible instead of $\gamma_{i}$ is justified: If we let $f(\beta, \gamma)=\frac{\gamma+2(\rho-1) \beta-1}{1+\beta}$, then

$$
\frac{\partial f}{\partial \gamma}=\frac{1}{1+\beta}<\frac{\partial f}{\partial \beta}=\frac{2(\rho-1)-\gamma+1}{(1+\beta)^{2}} \Leftrightarrow \beta+\gamma<2 \rho-1 .
$$

The latter, however, is true as we assume $\beta+\gamma \leq \rho-1$.)

Case 3: $q_{i+1}=q_{i}$.

Induction yields

$$
\frac{q_{i+1}}{p_{i+1}}=\frac{q_{i}}{p_{i+1}} \leq\left(1-\alpha_{i+1}\right) \frac{q_{i}}{p_{i}} \leq \frac{1-\alpha_{i+1}}{\hat{\rho}-1} .
$$


By Lemma 4 we can assume $\beta_{i}=0$, and thus

$$
\begin{aligned}
\Phi_{i+1} & =\gamma_{i}-1+(\rho-1) \frac{q_{i+1}}{p_{i}}=\gamma_{i}-1+(\rho-1) \frac{q_{i}}{p_{i}} \\
& \leq \gamma_{i}-1+(\rho-1) \frac{1}{\hat{\rho}-1} \leq \gamma_{i} \leq \rho-1 .
\end{aligned}
$$

To argue that $\alpha_{i+1} \leq 1 /(\hat{\rho}-1)$ we shift the concatenated rectangles $q_{i}, p_{i}$ down as far as possible, i.e., until either $\gamma_{i}=0$ or $\alpha_{i}=0$ (thereby increasing $\alpha_{i+1}$ ). By shifting $q_{i}, p_{i}$ down, the length of $p_{i}$ decreases, therefore $\gamma_{i}$ can become 0 . At the same time $p_{i+1}$ increases, causing the optimal and online solution to increase by the same amount. So the online algorithm is still $\rho$-competitive after this shift.

If $\gamma_{i}=0$, then $\alpha_{i+1} p_{i+1} \leq(\rho-1) q_{i+1}=(\rho-1) q_{i} \leq p_{i}$. Thus $\alpha_{i+1} \leq 1 / 2 \leq$ $1 /(\hat{\rho}-1)$.

If $\alpha_{i}=0$, the rectangles $p_{i-1}, q_{i}, p_{i}$ are concatenated. To show $\alpha_{i+1} \leq 1 /(\hat{\rho}-1)$ for this case, we distinguish three subcases.

Case 3a: $q_{i+1}=q_{i}=\alpha_{i-1} p_{i-1}$.

By Lemma 4 we can assume $\beta_{i-1}=0$ (and hence $p_{i}=p_{i-1}$ ). Thus we conclude that $\gamma_{i} p_{i}=\gamma_{i-1} p_{i-1}+(\rho-1) q_{i}-p_{i} \leq \gamma_{i-1} p_{i-1}$. Thus (using Lemma 1 again),

$$
\begin{aligned}
\alpha_{i+1} p_{i+1} & \leq(\rho-1) q_{i+1}+\gamma_{i} p_{i} \\
& \leq(\rho-1) \alpha_{i-1} p_{i-1}+\gamma_{i-1} p_{i-1} \\
& \leq(\rho-1) \alpha_{i-1} p_{i-1}+\left(\rho-1-\alpha_{i-1}\right) p_{i-1} \\
& \leq(\rho-1) p_{i-1}+(\rho-2) \alpha_{i-1} p_{i-1},
\end{aligned}
$$

and therefore

$$
\begin{aligned}
\alpha_{i+1} & =\frac{\alpha_{i+1} p_{i+1}}{p_{i}+\alpha_{i+1} p_{i+1}} \leq \frac{\rho-1+(\rho-2) \alpha_{i-1}}{\rho+(\rho-2) \alpha_{i-1}} \\
& \leq \frac{\rho-1+(\rho-2) /(\hat{\rho}-1)}{\rho+(\rho-2) /(\hat{\rho}-1)}<\frac{1}{\hat{\rho}-1}
\end{aligned}
$$

for $\rho \leq \hat{\rho}$.

Case $3 b: q_{i+1}=q_{i}=\beta_{i-1} p_{i-1}$.

$$
\begin{aligned}
\alpha_{i+1} p_{i+1} & \leq(\rho-1) q_{i+1}+\gamma_{i} p_{i}=(\rho-1) q_{i}+\gamma_{i} p_{i} \\
& \leq \text { by (2) }\left(\frac{(\rho-1)^{2}}{\rho}+\gamma_{i}\right) p_{i} .
\end{aligned}
$$

Since $\alpha_{i}=0$ and $\beta_{i}=0$ we have $\Phi_{i}=\gamma_{i} \leq\left(2(\rho-1)^{2}-1\right) / \rho$ (cf. (3)). Thus,

$$
\begin{aligned}
\alpha_{i+1} & =\frac{\alpha_{i+1} p_{i+1}}{p_{i}+\alpha_{i+1} p_{i+1}} \leq \frac{\frac{(\rho-1)^{2}}{\rho}+\gamma_{i}}{1+\frac{(\rho-1)^{2}}{\rho}+\gamma_{i}} \\
& \leq \frac{(\rho-1)^{2} / \rho+2\left((\rho-1)^{2}-1\right) / \rho}{1+(\rho-1)^{2} / \rho+2\left((\rho-1)^{2}-1\right) / \rho} .
\end{aligned}
$$


The latter is increasing in $\rho$, so we conclude (after multiplying the enumerator and denominator with $\rho$ ) that

$$
\alpha_{i+1} \leq \frac{(\hat{\rho}-1)^{2}+2\left((\hat{\rho}-1)^{2}-1\right)}{\hat{\rho}+(\hat{\rho}-1)^{2}+2\left((\hat{\rho}-1)^{2}-1\right)}=\frac{1}{\hat{\rho}-1}
$$

by definition of $\hat{\rho}$. (Recall that $3 \hat{\rho}^{2}-9 \hat{\rho}+4=0$.)

Case 3c: $q_{i+1}=q_{i}=q_{i-1}$. By Lemma 4 we may assume $\beta_{i-1}=0$, so that actually $q_{i-1}, p_{i-1}, q_{i}, p_{i}$ are concatenated and $p_{i}=p_{i-1}$. Since $\gamma_{i} p_{i}=\gamma_{i-1} p_{i-1}+$ $(\rho-1) q_{i}-p_{i}<\gamma_{i-1} p_{i-1}$, i.e., the improvement of $A$ upon $\rho$-competitiveness decreases, the value of $\alpha_{i+1}$ is smaller than $\alpha_{i}$ could at most be, thus, in particular, less than $1 /(\hat{\rho}-1)$.

Lemma $6 \Phi_{i+1} \leq \Phi_{i}-\frac{\hat{\rho}-\rho}{\hat{\rho}-1}$.

Proof By case distinctions:

Case 1: $q_{i+1}=\alpha_{i} p_{i}$.

By Lemma 4 we can assume $\beta_{i}=0$. Furthermore, Lemma 2 allows us to shift $p_{i+1}$ resp. the concatenated $q_{i+1} p_{i+1}$ down until $\alpha_{i+1}=\beta_{i+1}=0$ without affecting $\Phi_{i+1}$ (although this might result in a negative value of $\gamma_{i+1}$ in case $\Phi_{i+1}$ is negative). Summarizing, let us assume that $q_{i}, p_{i}, q_{i+1}, p_{i+1}$ are concatenated.

We seek to analyze how $\Phi_{i}$ and $\Phi_{i+1}$ vary as the concatenated $q_{i}, p_{i}, q_{i+1}, p_{i+1}$ are shifted upward. By Lemma 2, $\Phi_{i}$ remains unchanged. As to $\Phi_{i+1}$, observe that shifting $q_{i}, p_{i}, q_{i+1}, p_{i+1}$ upward by one unit will increase $\alpha_{i} p_{i}$ and hence $q_{i+1}$ as well as $p_{i}$ and $p_{i+1}$ by one unit each. Hence $A\left(L_{i+1}\right)$ increases by 4 . On the other hand, $O P T\left(L_{i+1}\right)$ increases by 2 , so that $\gamma_{i+1} p_{i+1}$ increases by $(2 \rho-4)$. Hence shifting $q_{i}, p_{i}, q_{i+1}, p_{i+1}$ upward will increase $\Phi_{i+1}$ as long as $\Phi_{i+1}<2 \rho-4$. So we are led to distinguish the following two cases:

$\Phi_{i+1}>2 \rho-4$ : In this case, $\Phi_{i+1}$ will increase as we shift $q_{i}, p_{i}, q_{i+1}, p_{i+1}$ downward. Doing so, we decrease $q_{i+1}$. So we eventually end up in a situation where either $q_{i+1}=q_{i}$ (which will be treated in Case 3 below) or $\gamma_{i}$ becomes zero (revealing that $\Phi_{i}=-(\rho-2) \alpha /(1-\alpha)$ must be negative $)$. But $\gamma_{i}=0$ implies

$$
\begin{aligned}
\gamma_{i+1} p_{i+1} & =\gamma_{i} p_{i}+(\rho-1) q_{i+1}-p_{i+1} \\
& =(\rho-1) \alpha_{i} p_{i}-p_{i+1} \leq(\rho-1) \alpha_{i} p_{i+1}-p_{i+1} .
\end{aligned}
$$

So $\Phi_{i+1}=\gamma_{i+1}=(\rho-1) \alpha_{i}-1<0$, contradicting our assumption that $\Phi_{i+1}>$ $2 \rho-4$.

$\Phi_{i+1} \leq 2 \rho-4$ : In this case $\Phi_{i+1}$ increases as we shift $q_{i}, p_{i}, q_{i+1}, p_{i+1}$ upward until either $q_{i}$ gets tight in the sense that $A\left(L_{i}-p_{i}\right)=\rho O P T\left(L_{i}-p_{i}\right)$ or $\Phi_{i+1}=$ $2 \rho-4$ is reached. The latter is impossible: Since $\gamma_{i+1} p_{i+1}=\gamma_{i} p_{i}+(\rho-1) q_{i+1}-$ $p_{i+1}$, we conclude (using Lemma 1) that

$$
\begin{aligned}
\Phi_{i+1} & =\gamma_{i+1} \leq \gamma_{i}+(\rho-1) \alpha_{i}-1 \leq \rho-1-\alpha_{i}+(\rho-1) \alpha_{i}-1 \\
& \leq(\rho-2)+\frac{\rho-2}{\hat{\rho}-1}<2 \rho-4,
\end{aligned}
$$

contradicting our assumption that $\Phi_{i+1}=2 \rho-4$. 
Finally, assume that $q_{i}$ gets tight. In this case, $\gamma_{i} p_{i}=\rho\left(p_{i}-p_{i-1}\right)-p_{i} \geq$ $\rho \alpha_{i} p_{i}-p_{i}$. So $\gamma_{i} \geq \rho \alpha_{i}-1$. Similarily, $\gamma_{i+1} p_{i+1}=\gamma_{i} p_{i}+(\rho-1) q_{i+1}-p_{i+1}$. Dividing by $p_{i+1}=p_{i}$, we obtain $\gamma_{i+1}=\gamma_{i}+(\rho-1) \alpha_{i}-1$. Hence

$$
\Delta \Phi=\Phi_{i+1}-\Phi_{i}=\gamma_{i+1}-\Phi_{i}=\gamma_{i}+(\rho-1) \alpha_{i}-1-\frac{\gamma_{i}-(\rho-2) \alpha_{i}}{1-\alpha_{i}}
$$

is maximized when $\gamma_{i}$ is as small as possible, i.e. $\gamma_{i}=\rho \alpha_{i}-1$. Thus

$$
\Delta \Phi \leq 2 \rho \alpha_{i}-\alpha_{i}-2-\frac{2 \alpha_{i}-1}{1-\alpha_{i}}=(2 \rho-1) \alpha_{i}-\frac{1}{1-\alpha_{i}} .
$$

This shows that $\Delta \Phi$ is indeed strictly negative since

$$
\alpha_{i}\left(1-\alpha_{i}\right) \leq \frac{1}{2} \cdot \frac{1}{2}=\frac{1}{4}<\frac{1}{2 \rho-1}
$$

for $\rho \leq \hat{\rho}$.

Case 2: $q_{i+1}=\beta_{i} p_{i}$.

By Lemma 3 we have

$$
\Delta \Phi=\Phi_{i+1}-\Phi_{i}=\frac{\gamma_{i}+2(\rho-1) \beta_{i}-1}{1+\beta_{i}}-\frac{\gamma_{i}+\beta_{i}-(\rho-2) \alpha_{i}}{1-\alpha_{i}} .
$$

The derivative with respect to $\gamma_{i}$ of the above is $1 /\left(1+\beta_{i}\right)-1 /\left(1-\alpha_{i}\right) \leq 0$, so $\Delta \Phi$ is decreasing in $\gamma_{i}$. Thus we may choose $\gamma_{i}=0$. Additionally, we have $\alpha_{i} \leq \beta_{i}$, otherwise we are not in this case. With $\gamma_{i}=0$ and under the constraints $\alpha_{i} \leq \beta_{i} \leq$ $\rho-1, \alpha_{i}+\beta_{i} \leq \rho-1$ we have

$$
\Phi_{i+1}-\Phi_{i}=\frac{2(\rho-1) \beta_{i}-1}{1+\beta_{i}}-\frac{\beta_{i}-(\rho-2) \alpha_{i}}{1-\alpha_{i}}<-0.04 .
$$

Indeed, the function

$$
\Delta=\Delta(\alpha, \beta)=\frac{2(\rho-1) \beta-1}{1+\beta}-\frac{\beta-(\rho-2) \alpha}{1-\alpha}
$$

has partial derivative $\Delta_{\alpha}=0$ for $\beta=\rho-2$. Plugging this value into $\Delta$, we find that the resulting $\Delta$ is independent of $\alpha$ and equals $\rho-2-\frac{1}{\rho-1}<-0.1$. On the boundary $\alpha=0$ the function $\Delta=\Delta(\beta)$ is less than -0.04 for $\beta \in[0, \rho-1]$ and on the boundary $\alpha=\beta$ the function $\Delta=\Delta(\beta)$ is bounded from above by -0.2 for $\beta \in\left[0, \frac{\rho-1}{2}\right]$. (Note that $\alpha=\beta$ implies $\beta \leq \frac{\rho-1}{2}$.) We omit the details.

Case 3: $q_{i+1}=q_{i}$.

By definition of $q_{i+1}$, we have $\alpha_{i} p_{i} \leq q_{i+1}=q_{i}$. Thus, Lemma 5 implies $\alpha_{i} \leq$ $q_{i} / p_{i} \leq \frac{1-\alpha_{i}}{\hat{\rho}-1}$ or, equivalently, $\alpha_{i} \leq 1 / \hat{\rho}$. Thus we conclude that

$$
\Phi_{i}=\frac{\gamma_{i}-(\rho-2) \alpha_{i}}{1-\alpha_{i}} \geq \frac{-(\rho-2) \alpha_{i}}{1-\alpha_{i}} \geq \frac{-(\rho-2) / \hat{\rho}}{1-1 / \hat{\rho}}>\hat{\rho}-3
$$

for $\rho \leq \hat{\rho}$. 
Now, as in Case 1, let us assume that $\beta_{i}=\beta_{i+1}=0$ and shift the concatenated $q_{i+1} p_{i+1}$ down until $\alpha_{i+1}$ becomes zero as well. This leaves $\Phi_{i+1}$ (and, of course, also $\left.\Phi_{i}\right)$ invariant, but may result in a negative value of $\gamma_{i+1}$ in case $\Phi_{i+1}\left(=\gamma_{i+1}\right.$ after the shift) is negative. (As we are only interested in $\Delta \Phi$, we do not care about negative values of $\gamma_{i+1}$ here.) Thus assume that $q_{i}, p_{i}, q_{i+1}, p_{i+1}$ are concatenated. We claim that shifting the concatenated $q_{i}, p_{i}, q_{i+1}, p_{i+1}$ down increases $\Phi_{i+1}$ (while leaving $\Phi_{i}$ unchanged). Indeed moving down decreases both $p_{i}$ and $p_{i+1}$ by one unit, so that in total $A\left(L_{i+1}\right)$ decreases by 3 units, while $O P T\left(L_{i+1}\right)$ decreases by only one unit. Thus $\gamma_{i+1} p_{i+1}$ increases with $3-\rho$ units while $p_{i+1}$ decreases with one unit. This shows that moving $q_{i}, p_{i}, q_{i+1}, p_{i+1}$ down increases $\Phi_{i+1}=\gamma_{i+1}$ whenever $\Phi_{i+1}>\rho-3$.

To show that $\Phi$ decreases, we may assume that $\Phi_{i+1}>\rho-3$-else a significant decrease of at least $\hat{\rho}-\rho$ follows already immediately from (4). But then, as we have seen above, we may also assume w.l.o.g. that $q_{i}, p_{i}, q_{i+1}, p_{i+1}$ is shifted down as far as possible, i.e., until $\alpha_{i}=0$. (Note that this may even result in a negative value of $\gamma_{i}$, but we do not care, as we are only interested in $\Phi$-values.) When $\alpha_{i}=0$, however, then

$$
\Delta \Phi=\Phi_{i+1}-\Phi_{i}=\gamma_{i+1}-\gamma_{i}
$$

is significantly negative since $\gamma_{i+1} p_{i+1}=\gamma_{i} p_{i}+(\rho-1) q_{i+1}-p_{i+1}$, so that $\gamma_{i+1} \leq$ $\gamma_{i}+\frac{\rho-1}{\hat{\rho}-1}-1$.

Summarizing, in each case there is a significant decrease in the potential function, provided that $\rho<\hat{\rho}$.

\section{Conclusions}

We have solved the (30 year old) problem of analysing Brown-Baker-Katseff sequences and provided an optimal online algorithm for these sequences. Up to now all previous lower bounds for online strip packing were based on Brown-Baker-Katseff sequences. A natural question to ask is whether (or to what extent) these sequences are worst case sequences for online strip packing. In Harren and Kern (2011) we have tried to analyze more generally sequences of "thin" items $p_{i}$ and "blocking" items $q_{j}$, which are not necessarily alternating. We suceeded in proving lower and upper bounds close to 2.6 for such sequences. Determining the exact value appears to be involved, but in any case, our results show that to close the gap between the currently best lower bound of 2.6 and the currently best upper bound of 6.6 , completely new ideas are needed.

Acknowledgement Part of this research has been funded by the Dutch BSIK/BRICKS project.

Open Access This article is distributed under the terms of the Creative Commons Attribution License which permits any use, distribution, and reproduction in any medium, provided the original author(s) and the source are credited. 


\section{References}

Baker BS, Coffman EG, Rivest RL (1980) Orthogonal packings in two-dimensions. SIAM J Comput 9:846-855

Brown DJ, Baker BS, Katseff HP (1982) Lower bounds for on-line two-dimensional packing algorithms. Acta Inform 18:207-225

Fuchs B, Hochstaettler W, Kern W (2005) Online matching on a line. Theor Comput Sci 332(1-3):251264

Hurink JL, Paulus JJ (2008a) Online scheduling of parallel jobs on two machines is 2-competitive. Oper Res Lett 36:51-56

Hurink JL, Paulus JJ (2008b). Online algorithm for parallel job scheduling and strip packing. In: WAOA 2007. Lecture notes in computer science, vol 4927, pp 67-74

Harren R Kern, W (2011, to appear). Improved lower bound for online strip packing. In: Proceedings WAOA 2011. Springer lecture notes in computer science

Johannes B (2006) Scheduling parallel jobs to minimize the makespan. J Sched 9:433-452

Kern W (2010) A tight analysis of Brown-Baker-Katseff sequences for online strop packing (extended abstract). In: Proceedings of the 9th CTW 2010, pp 109-110

Pruhs K, Sgall J, Torng E (2004) Online scheduling. In: Leung JY-T (ed) Handbook of scheduling: algorithms, models, and performance analysis

Ye D, Han X, Zhang G (2009) A note on online strip packing. J Comb Optim 17(4):417-423 